\title{
Hydrodynamic Flow from Fast Particles
}

\author{
Jorge Casalderrey-Solana*† \\ Department of Physics and Astronomy, State University of New York, Stony Brook, NY 11794 \& \\ Lawrence Berkeley National Laboratory - 1 Cyclotron Rd, Berkeley, CA 94720 \\ E-mail: JCasalderrey-Solana@lbl.gov
}

\begin{abstract}
We study the hydrodynamical response of the quark gluon plasma to the passage of high momentum particle within the context of linearized hydrodynamics in a static fluid. Two modes can be excited, the sound and the diffuson. Assuming that significant part of the energy lost by the particle thermalizes we discuss the modification of the azimuthal dihadron distributions due to the jet induced flow (conical flow). If the energy loss is large and no significant entropy is produced, large angle correlations are produced at $\Delta \phi=\pi-1.2 \mathrm{rad}$. We also estimate the effect of the expanding fireball on the sound wave amplitude, which grows as a consequence of the dilution of the background medium. Finally, if the speed of sound goes to zero at the mixed phase (first order phase transition) reflected waves appear that lead to correlation at $\Delta \phi=1.4 \mathrm{rad}$.
\end{abstract}

The 2nd edition of the InternationalÂăWorkshop - Correlations and Fluctuations in Relativistic Nuclear Collisions -

July 7-9 2006

Galileo Galilei Institute, Florence, Italy

\footnotetext{
* Speaker.

${ }^{\dagger}$ In collaboration with E. V. Shuryak and D. Teaney.
} 


\section{Introduction}

Jet Quenching, the suppression of high momentum particles, is one of the major findings at RHIC [1]-帊. This strong suppression is due to the energy loss of partons traversing the hot and dense matter formed in ultrarelativistic heavy ion collisions. Different mechanisms such as radiative [5-8], collisional [9] and ionization [10] losses have been proposed to explain the observed suppression.

On the other hand, the bulk of matter formed at RHIC can be successfully described by ideal hydrodynamics [11, 12] and exhibits very strong collective effects such as radial and elliptic flow [13, 14]. The study of viscous effects in these flows [15] indicate that the viscosity of the Quark Gluon Plasma at RHIC is very small, close to the minimal conjectured bound [16]. This fact, among others, has motivated the suggestion that the QGP formed at RHIC is strongly coupled (sQGP) [17].

The good hydrodynamic behavior of the QGP indicates that the modification of matter as a consequence of the jet passage induces collective effects [18]. Under the assumption that most of the energy lost by the jet is shared among the matter constituents and eventually thermalized, we suggested [19, 20] that a conical flow appears as a consequence of the jet-medium interaction. This flow can be understood as sound emission from the jet. Since the medium speed of sound is smaller than the speed of the particle propagation, a Mach shock wave is formed (see Fig. 1).

The appearance of conical flow manifests itself in the modification of the dihadron distributions associated to a high $p_{T}$ hadron. The trigger hadron is produced by the fragmentation of a parton that, due to the strong quenching, is mainly produced at the surface of the interaction region. This biases the backjet to travel along the medium, loosing energy and inducing the Mach shock wave. At freeze out, the energy transferred to the sound wave is converted into particles, which are emitted in the Mach direction

$$
\cos \theta_{M}=c_{s}
$$

The experimental correlations measured by the PHENIX [21] and STAR [22] collaborations peak at an azimuthal angle $\Delta \phi=\pi-1.2 \mathrm{rad}$ with respect to the trigger particle, which agrees with the expectations from the picture described above.

In this work we will present our analysis based on linearized hydrodynamics on a static medium [19, 20] as well as in a medium with a variable speed of sound [23]. The study of flow effects on the sound propagation was performed in [24]. An attempt to include a realistic medium has been made in [25] and the non linear hydrodynamic response to the jet was studied with $2+1$ dimensional hydro code [26]. Transport model calculations have also studied the modification of the dihadron distributions, which are in agreement with the formation of conical flow [27].

Other sources of large angle correlations, in which the lost energy is not deposited in the medium, have been suggested. A similar Mach-like front can be formed from the plasmon [28] or from the transverse modes of the gauge fields [29-31] (Cerenkov radiation), as long as the speed of propagation of the corresponding mode in plasma is smaller than one. Large angle medium induced radiation has been also studied in [32, 33]. In [33] it has been shown that demanding that only one gluon is radiated from the propagating parton by the inclusion of a Sudakov factor leads to a radiated gluon distribution which is similar to the experimentally observed dihadron distribution. 


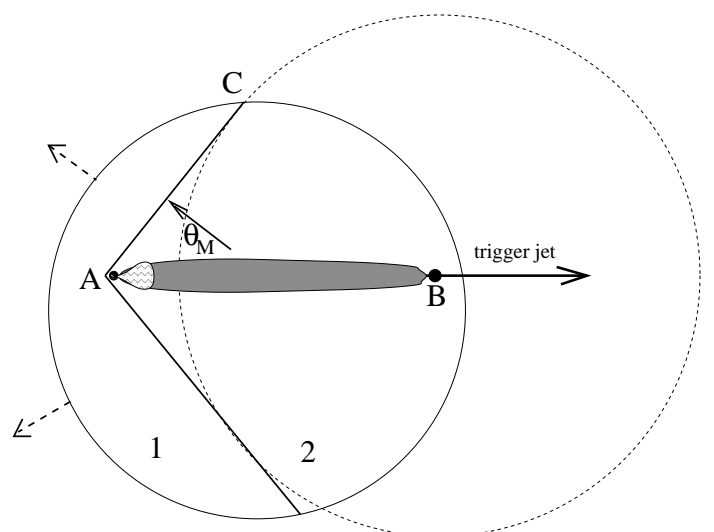

Figure 1: A schematic picture of the flow created by a jet moving through the fireball. The trigger jet is moving to the right away from the origination point (the black circle at point B). Sound waves start propagating as spherical waves (the dashed circle) from the origination point. The companion quenched jet is moving to the left creating a wake of matter (shaded area). The head of the jet is a non-equilibrium gluonic shower formed by the original hard parton (black dot A). The solid arrow indicates the flow velocity which is perpendicular to the shock cone at the angle $\theta_{M}, \cos \left(\theta_{M}\right)=c_{s} / c \simeq 0.55$.

Finally, in [34] it is argued that the deflection of the leading parton by the medium may explain the observed modifications.

\section{Interplay of Jets and Medium}

The passage of a high momentum particle through the quark gluon plasma leads to a modification of the local properties of the medium. This modification changes its hydrodynamic evolution and can be described by adding a source to the conservation equation:

$$
\partial_{\mu} T^{\mu v}=J^{v}
$$

The source $J^{\mu}$ encodes the medium excitation and thermalization process. From Eq. (2.1) it is easy to see that the total 4-momentum transferred to the hydro fields is given by

$$
\frac{d P^{\mu}}{d t}=\int d^{3} x J^{\mu}
$$

Unfortunately, the constraint Eq. (2.2) is not enough to specify the source. ${ }^{1}$ As we will see in section 4 , different sources that fulfill Eq. (2.2) lead to very different observable consequences.

The space time dependence of the source $J$ is ultimately determined by the microscopic mechanism that describes the jet-medium coupling, which is unknown. Since the initial modification of the hydrodynamic fields is large [19], we expect a complicated functional form for this source.

In this work we study, in a static background, the different types of flows compatible with Eq. (2.2) without specifying a source. In section 3, by analyzing the induced flow far from the jet we discover two types of excitation mechanisms. Their observable consequences are studied in

\footnotetext{
${ }^{1}$ the addition of any total derivative to $J$ would also satisfy Eq. (2.2)
} 
section $\sharp$ by specifying sources compatible with the different mechanisms. In section 5 we study some expansion effects and we finish with some conclusions.

\section{Excitation Mechanisms}

Regardless of the violent initial hydrodynamic disturbance, far from the interaction region the perturbation is described by linearized hydrodynamics. In this region, where the source vanishes, the evolution equation Eq. (2.1) splits into two modes:

Sound: propagating mode with an irrotational velocity field (v) which can be described via a scalar potential $\varphi$ such that $T \mathbf{v}=\nabla \varphi$ [35]. Its evolution equation is

$$
\partial_{0}^{2} \varphi-c_{s}^{2} \nabla^{2} \varphi-\Gamma_{s} \partial_{t} \nabla^{2} \varphi=0 .
$$

Diffuson: non propagating mode in which the velocity field, $R^{i}$, is rotational and which is governed by the equation

$$
\partial_{0} R_{i}=\frac{3}{4} \Gamma_{s} \nabla^{2} R_{i}
$$

In Eq. (3.1) and Eq. (3.2) $\Gamma_{s}=4 \eta / 3 T s$ is the sound attenuation length, with $\eta$ the shear viscosity $T$ the temperature and $s$ the entropy density of the fluid.

To proceed further, we consider a parton moving in a static QGP with a fixed velocity $v$ along the $-\hat{x}$ direction. We assume that the parton has traveled for a long time such that a stationary situation has been reached. With this assumption, the solutions for Eq. (3.1) and Eq. (3.2) are

$$
\begin{aligned}
\varphi & =\frac{T}{2 \pi s} \int_{-\infty}^{x+v t-\beta \rho} d \xi \frac{d F / d x(\xi)}{\sqrt{(x+v t-\xi)^{2}-\beta^{2} \rho^{2}}}, \\
R^{x} & =\frac{A}{\left(2 \pi \frac{3}{2 v} \Gamma_{s}(x+v t)\right)} \exp \left\{-\frac{\rho^{2}}{\left(\frac{3}{2 v} \Gamma_{s}(x+v t)\right)}\right\},
\end{aligned}
$$

where $\rho$ is the transverse distance to the jet and $\beta^{2}=v^{2} / c_{s}^{2}-1$. In these expressions $d F / d x(\xi)$ and $A$ depend on the source. The function $d F / d x(\xi)$ can be interpreted as the flux of entropy per unit length out of an infinite cylinder surrounding the jet. The constant $A$ can be related to the total entropy produced by the interaction/thermalization process Eq. (3.8).

A sketch of the velocity field along the jet direction can be seen in Fig. 2. The sound waves are concentrated along the Mach direction Eq. (1.1) and propagate to long transverse distances from the jet. The diffuson field, directed towards the jet propagation, is concentrated along the jet axes within a transverse size $\Sigma_{0}$ which grows as the square root of the distance to the jet position.

We compute now the energy and momentum carried away by the flow in the parton rest frame. Since in this frame the fields are static, we compute the 4-momentum flux out of a long cylinder surrounding the jet. This is the energy and momentum lost by the jet which incorporates into the evolution of the fluid. Denoting by $C$ a long cylinder surrounding the jet and $\Sigma$ the end cup to the right of jet, the energy lost per unit proper time is given by,

$$
-\frac{d E_{j}}{d \tau}=\int_{C}\left(T^{0 \rho}\right)^{\prime}+\int_{\Sigma-\Sigma_{0}}\left(T^{0 \chi}\right)^{\prime}+\int_{\Sigma_{0}}\left(T^{0 \chi}\right)^{\prime}
$$




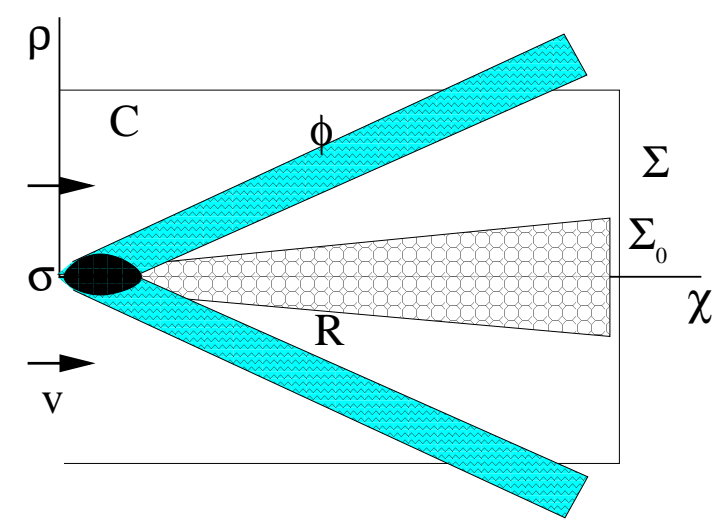

Figure 2: Sketch of the flow picture in the jet rest frame. The sound component of the velocity field (wavy region) propagates out of the source and leads to a small disturbance at large distances. The diffuson part(circled region) remains concentrated along the jet axis within a transverse size that grows as $\sqrt{\chi}$.

where we have separated the contributions of the sound (the first two terms) and the diffusion mode. A similar expression can be found for the momentum loss and for the entropy production in the jet medium interaction. In fact, without loss of generality, the energy lost and the entropy production can be related by means of their relation to the potential $\varphi$ Eq. (3.3),

$$
-\frac{d E_{j}}{d \tau}=T \gamma \frac{d S_{j}}{d \tau}-\frac{d E_{j}^{R}}{d \tau}-T \gamma \frac{d S_{j}^{R}}{d \tau}
$$

where $d E_{j}^{R} / d \tau, d S_{j}^{R} / d \tau$ are the energy and entropy rates carried by the diffuson mode.

To this point we have not made any assumption about the energy and momentum deposited by the jet. From now on, we will assume that the particle propagates almost on shell, which means that the energy and momentum in the fluid rest frame are linked by $d E / d t=v d P / d t$, which in the jet rest frame means

$$
\frac{d E_{j}}{d \tau} \approx 0
$$

From Eq. (3.6) and Eq. (3.7) we conclude that if the interaction mechanism produces significant entropy, then rotational flow is needed. Since $d E_{j} / d \tau=0$, the entropy production only depends on the amplitude of the diffuson mode,

$$
\frac{1}{\gamma} \frac{d S_{j}}{d \tau}=\frac{1}{\gamma} \frac{d S_{j}^{R}}{d \tau}+\frac{1}{T \gamma^{2}} \frac{d E_{j}^{R}}{d \tau}=v^{2} s A
$$

Thus, we can distinguish two different types of medium excitations:

1. Isentropic excitations. The interactions of the jet and the fluid are such that no entropy is produced in the process. In this case the energy/momentum deposited can be calculated from the the far field sonic wave and it is quadratic in the perturbation.

2. Non isentropic excitations. The main mechanism of energy deposition proceeds by transferring of heat into the fluid, creating new entropy. As a consequence, rotational flow is needed, which is concentrated in a narrow wake behind the fluid. 


\section{Observable Consequences}

In this section we study the effect of the two kinds of medium excitations identified in the previous section. To do so, we need to specify a source compatible with the classification above. In the linearized region, the modified momentum densities are

$$
T^{\prime 0 \mu}(\mathbf{x}, t)=\int d t_{0} \boldsymbol{\theta}\left(t-t_{0}\right) \delta T^{0 \mu}\left(\mathbf{x}-\mathbf{v} t_{0}, t-t_{0}\right),
$$

where $\delta T^{0 \mu}\left(0, \mathbf{x}-\mathbf{v} t_{0}\right)$ is the modification in an infinitesimal displacement of the particle between $t$ and $t+d t$. From Eq. (2.1) and Eq. (4.1), the source which corresponds to this modification is

$$
J^{\mu}(\mathbf{x}, t)=\delta T^{0 \mu}(\mathbf{x}-\mathbf{v} t, 0) .
$$

From these modified fields we compute the spectrum of particles associated with the jet by means of the Cooper-Frye prescription. We use an equal time freeze out condition,

$$
\frac{d N}{d^{3} p}=\int_{V} \frac{d^{3} V}{2 \pi^{3}} e^{-\frac{E}{T}+\delta}, \quad \delta=\frac{E}{T} \frac{\delta T}{T}+\frac{\mathbf{p v}}{T},
$$

where $\mathrm{V}$ is the volume of the fireball, $\mathbf{v}$ the velocity field and $\mathrm{E}$ and $\mathbf{p}$ are the energy and momentum of the associated particle.

Regardless of the excitation mechanisms, Eq. (4.3) indicates that the spectrum of low momentum particles is independent of the flow field. In fact, expanding the exponent we obtain,

$$
\frac{d N}{d^{3} p}=\frac{e^{-\frac{E}{T}}}{(2 \pi)^{3}}\left(V+\frac{E}{T} \frac{E_{d e p}}{T s}+\frac{\mathbf{P}}{T} \frac{\mathbf{P}_{d e p}}{T s}\right)
$$

where $E_{d e p}$ and $\mathbf{P}_{d e p}$ are the total energy and momentum deposited by the jet. Thus, the only correlation we expect at low momentum is a $\cos (\Delta \phi)$ of the relative angle between the momentum of the particle and the back jet. At high momentum, on the contrary, the spectrum reflects the flow field and the different excitation mechanism lead to different correlation functions.

\subsection{Isentropic Excitations}

As discussed in section 3, isentropic excitations are those in which only the sound mode is excited. This is achieved by the following initial modification of the momentum densities, ${ }^{2}$

$$
\delta T^{00}(\mathbf{x}, 0)=0, \quad \delta T^{0 i}(\mathbf{x}, 0)=\frac{C}{\sigma^{4}} \partial_{i} e^{-r^{2} / 2 \sigma^{2}},
$$

where $\sigma$ is the typical size in which the linearized theory is applicable, and $\mathrm{C}$ is the (dimensionless) amplitude. The far field solution that corresponds to this source is Eq. (3.3) with

$$
\frac{d F}{d x}(\xi)=-\frac{2 \pi}{T} \frac{v^{2}}{c_{s}^{3}} \frac{C}{\bar{\sigma}^{2}} \partial_{\xi} e^{-\xi^{2} / 2 \bar{\sigma}^{2}}
$$

\footnotetext{
${ }^{2}$ Note that from Eq. (4.2) and Eq. (2.2) one may conclude incorrectly that this choice carries no energy nor momentum. Eq. (4.2) is based is the first order relation Eq. (4.1), while the momentum losses are second order Eq. (4.7).
} 

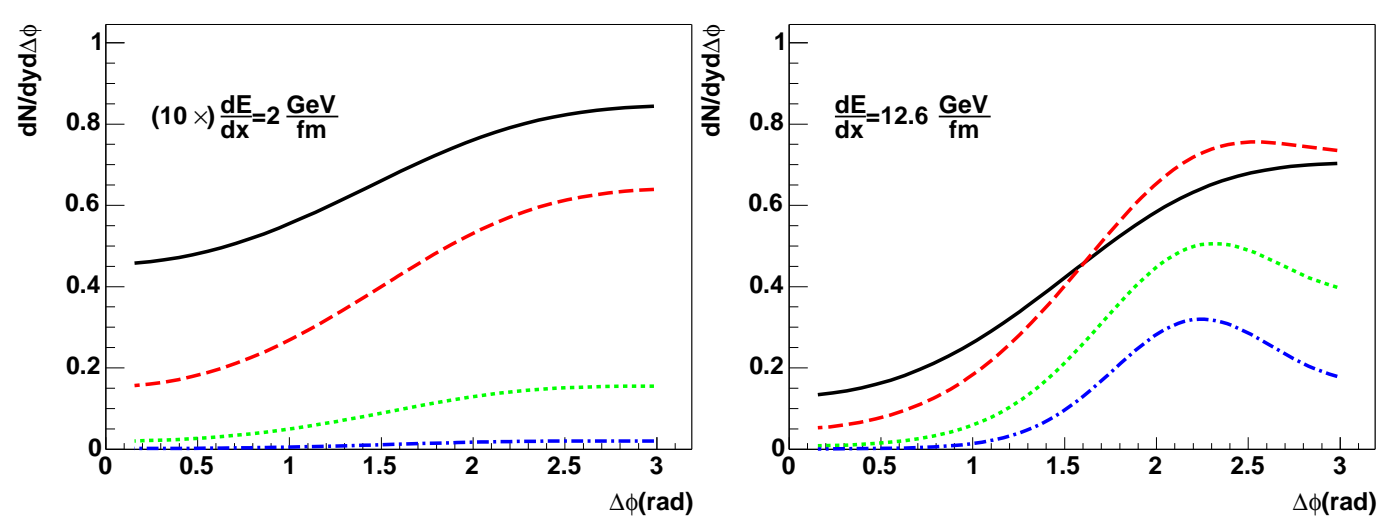

Figure 3: Isentropic Excitations.Associate yield dependence on associate $p_{T}$ for fixed source size $\sigma=$ $0.75 / T$, viscosity $\Gamma_{s}=0.1 / T, t_{j}=8 / T, t_{f}=10 / T$, and energy loss, $d E / d x=10 T^{2}$ (left) and $d E / d x=$ $63 T^{2}$ (right). The label values for $d E / d x$ correspond to $T=200 \mathrm{MeV}$. The three curves are for $1 T<p_{t}<5 T$ (solid), $5 T<p_{t}<10 T$ (dotted), $(3 \times) 10 T<p_{t}<15 T$ (dashed), $(10 \times) 15 T<p_{t}<20 T$ (dashed-dotted). (in the upper panel all the curves are rescaled further up by a factor 10). No large angle correlation is observed for $d E / d x=10 T^{2}$. For $d E / d x=63 T^{2}$ the position of the peak shifts toward $\pi$ for lower $p_{T}$.

where we have defined $\bar{\sigma}=v \sigma / c_{s}$. Performing a similar analysis to that of section B, the energy/momentum loss is given by [19]

$$
\begin{aligned}
\frac{d E}{d t} & =-v \frac{d P^{x}}{d t}=-v \frac{d P_{j}^{x}}{d \tau} \\
\frac{d P_{j}^{x}}{d \tau} & =-\frac{1}{4 \pi} \frac{T}{s} \int_{-\infty}^{\infty} d \xi_{1} d \xi_{2} \frac{d}{d \xi} d F / d x\left(\xi_{1}\right) \frac{d}{d \xi} d F / d x\left(\xi_{2}\right) \ln \left|\xi_{1}-\xi_{2}\right| .
\end{aligned}
$$

This expression is well know in non relativistic fluid dynamics [35]. For the source Eq. (4.5),

$$
\frac{d E}{d x} \approx \frac{\pi^{2}}{v^{2}} \frac{1}{s T} \frac{C^{2}}{\sigma^{6}}
$$

The energy deposited into the fluid cannot fix the parameters of the source. We will fix the value of $\sigma=0.75 / T$ and determine the amplitude from the energy loss. Note that $d E / d x$ is quadratic in the amplitude of the perturbation.

In Fig. 3 we show the number of correlated particles for different associate $p_{T}$ ranges for two different values of the energy loss, $d E / d x=10 T^{2}$ (left) and $d E / d x=63 T^{2}$ (right). For a typical temperature of $T=200 \mathrm{MeV}$ these correspond to $d E / d x=2 \mathrm{GeV} / \mathrm{fm}$ and $d E / d x=12.6 \mathrm{GeV} / \mathrm{fm}$. The jets are assumed to travel in the medium for $t_{j}=8 / T$ and the freeze out is at $t_{f}=10 / T$.

At moderate values of the energy loss, the perturbation is not large enough to reflect the Mach Cone in the spectrum, and the correlation function is that of low momentum particles, Eq. (4.4). For larger values of the energy loss, the low momentum particles also follow the expected behavior, Eq. (4.4), but as the momentum of the associated particle increases the maximum of the correlations shifts to the left and saturates at $\Delta \phi=\pi-\theta_{M}$. This qualitative behavior seems to be supported by the experimental data. In fact, the experimental dihadron azimuthal distributions in which the associated particle is hard $\left(1<p_{t}<2.5 \mathrm{GeV}\right)$, as those in [21], show a large angle correlation at $\Delta \phi=\pi-1.2$ which corresponds to our expectations. On the other hand, those dihadron distribution 


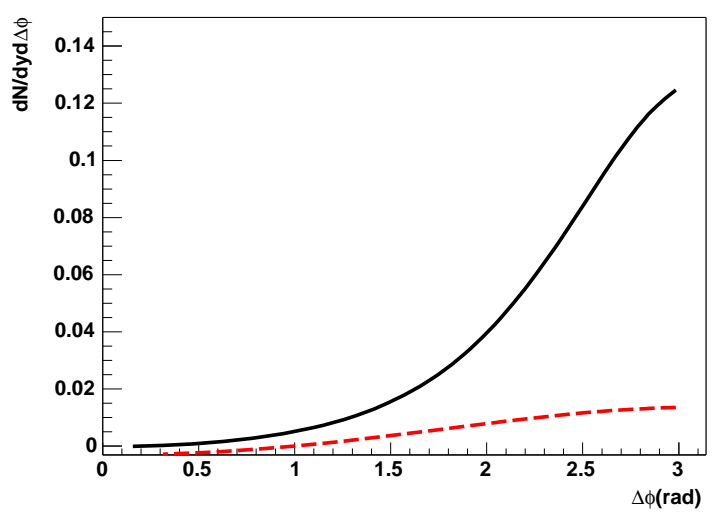

Figure 4: Non Isentropic excitations. Associate yield dependence for fixed source size $\sigma=0.75 / T$, viscosity $\Gamma_{s}=0.1 / T, t_{j}=8 / T, t_{f}=10 / T$. The $p_{T}$ interval is $10 T<p_{T}<20 T$. The two curves correspond to energy loss, $(5 \times) d E / d x=10 T^{2}$ (dashed) and $d E / d x=63 T^{2}$ (solid). For $T=200 \mathrm{MeV}$, these correspond to $d E / d x=2,12.6 \mathrm{GeV} / \mathrm{fm}$ respectively. No large angle correlations are observed.

dominated by softer particles $\left(0.15<p_{t}<4 \mathrm{GeV}\right)$ [22] show a much broader distribution, which is consistent with the low momentum behavior observed in Fig 3 .

Even though the large values required for the energy loss could be supported from the large $\hat{q}$ extracted from experimental fits [36], it is not phenomenologically acceptable that a typical jet travels through such an opaque medium for a time long enough to develop the shock. As we will see in section 5, expansion effects are crucial for this matter.

\subsection{Non Isentropic Excitations}

In this kind of excitations both the sound and the diffuson modes are excited. A source that leads to this kind of excitation is

$$
J^{\mu}=\frac{d E}{d x} \frac{e^{-\left(\mathbf{x}-\mathbf{r}_{\mathbf{j}}(t)\right)^{2} / 2 \sigma^{2}}}{\left(2 \pi \sigma^{2}\right)^{3}} \cdot(1,-1,0,0),
$$

where, as before, we take $\sigma=0.75 / T$. As opposed to the previous case, the amplitude of the perturbation is linear in the energy loss (and independent of $\sigma$ )

Fig 4 shows the correlation function obtained for this source. We show the result for two different values of the energy loss $d E / d x=10 T^{2}$ and $d E / d x=63 T^{2}$. As opposed to the isentropic case, there is no large angle correlation for any of the energies studied. This is a consequence of the excitation of the diffusion mode.

As we discussed in section 3 , the appearance of rotational flow makes matter to move preferentially along the jet direction. Thus, even though sound waves are produced in the medium, they are not observed in the final correlation function. ${ }^{3}$

\footnotetext{
${ }^{3}$ The source studied in [26] is of the kind of Eq. (4.9). This is why no large angle correlations are observed as a result of the jet induced flow.
} 


\section{Expansion Effects}

The discussion of the previous sections focused on a static medium. We now study the behavior of the amplitude of the Mach shock in a expanding medium as well as the effect of a variable $c_{s}$ [23]. The influence of the directionality of the underlying flow was studied in [24].

\subsection{Amplitude Increase}

Since all our analysis is based on linearized hydrodynamics, we study a simple model for the expansion, that of static fluid in an expanding universe (Big Bang like). For definiteness, we use the flat Robertson-Walker metric

$$
d \tau^{2}=d t^{2}-R(t)^{2}\left[d r^{2}+r^{2}\left(d \theta^{2}+\sin ^{2} \theta d \phi\right)\right],
$$

where the parameter $R(t)$ (the instantaneous Hubble radius of our "universe") is treated as external.

The linearized hydro equations in this space are easily expressed in terms of the variable $G^{i}=R^{5} T^{\prime 0 i}$. For sound, where the velocity field is a gradient, its space Fourier transformed is

$$
\mathbf{G}(t, \mathbf{k})=i \mathbf{k} G(t, \mathbf{k}) .
$$

The evolution equation of $G$ is that of a harmonic oscillator with variable mass and frequency,

$$
\partial_{\eta}\left(\bar{M} \partial_{\eta} G\right)+\bar{M} \bar{\omega}^{2} G=0,
$$

where $d \eta / d t=1 / R, \bar{\omega}=k c_{s}$ and $\bar{M}=M / c_{s}^{2}$ with

$$
\frac{1}{M} \frac{d M}{d \eta}=\left(\left(3 c_{s}^{2}-1\right) \frac{1}{R} \frac{d R}{d \eta}\right) \Longrightarrow M=\frac{1}{T R} .
$$

In open mechanical systems with slowly varying parameters there is an approximate constant of motion, the adiabatic invariant I [37]. In the case of a harmonic oscillator $I=<H>/ \omega$, where $H$ is the Hamiltonian and the average is taken over phase space cycles.

In the particular case at hand, we use the adiabatic invariant to predict the variation of the amplitude of the sound wave. Since the spectrum Eq. (4.3) depends on $v / T$, the relevant quantity is the ratio of the velocity field amplitude for a fixed $k, v_{k}$, to the T. A simple manipulation leads to

$$
\frac{v_{k}}{T}=\sqrt{2 R^{3} T \bar{\omega} I} .
$$

From Eq. (5.5) and Eq. (5.4) we conclude, that as long as $c_{s}^{2}<1 / 3$, if the medium expands the relevant amplitude for the observation of the conical flow increases. In spite of the fact that our model does not describe the fireball at RHIC we can use this formula to get an estimate of the effect. From hydrodynamic simulations [11, 12] we estimate the changes of the system size, temperature and $c_{s}$ and we obtain a factor 3 increase of this quantity.

The increase by a factor of 3 of the relevant amplitude is crucial for the observation of the conical flow. Thus, a wave in an expanding medium needs to have an amplitude 3 times smaller than that in a static background to produce the same observable effects (since the amplitude grows by the expansion). Since for the isentropic excitations the energy loss is quadratic in the amplitude of the wave, the required energy loss in an expanding medium is 9 times smaller than in a static one, which leads to a more phenomenologically acceptable value. 


\subsection{Variable Speed of Sound}

The matter created in an relativistic heavy ion collision at RHIC passes through three different phases during its evolution: the QGP phase, where the speed of sound is $c_{s}^{Q G P}=1 / \sqrt{3}$; the hadron gas, with $c_{s}^{H G}=\sqrt{0.2}[38,39]$; and the mixed phase, where the speed of sound has a minimum close to zero.

Since the speed of sound determines the position of the peak, such a change is reflected in the particle production. It is possible to give a simple estimate of this effect by following the position of the shock. From hydrodynamic simulations [11, 12] we conclude that the duration of the three stages is comparable and of the order $t_{M} \sim 4 \mathrm{fm}$. Thus we expect angular correlations at an angle

$$
\cos \theta_{M}=\bar{c}_{s}=1 / \tau \int_{o}^{\tau} d t c_{s}(t) \approx 0.3 \Longrightarrow \Delta \phi \approx \pi-1.2,
$$

which agrees with the peak position in the experimental dihadron distributions [21, 22].

The dropping of the speed of sound leads to another interesting effect. If the speed of sound goes to zero during the mixed phase, as it does in a first order phase transition, a reflected wave appears at the end of this phase. In [23] we demonstrate it explicitly within the simplified model for the expansion outlined above. The relative amplitude of the reflected wave with respect to the transmitted one depends on how close $c_{s}$ is to zero. What is more, since $c_{s}^{Q G P}$ in the QGP is larger than $c_{s}^{H G}$, and the times of both phases are comparable, the freeze out finds the reflected wave moving backwards from the transmitted one.

If the QCD phase transition is first order, the interference of the reflected waves leads to the formation of a second shock moving in the direction of the triggered particle. Its directionality can be estimated as we did for the transmitted front. Before the mixed phase the front moves a distance $A B=c_{S}^{Q G P} t_{M}$. During the mixed phase the wave stalls, resuming its motion in the hadron gas phase, where it moves backwards a smaller distance $B C=c_{s}^{H G} t_{M}$. At freeze out, the shock front is at $A C=A B-B C$ and since reflected waves are emitted before the mixed phase

$$
\cos \left(\theta_{e}\right) \approx \frac{A C}{t_{M}} \Longrightarrow \theta_{e} \approx 1.4 \mathrm{rad} .
$$

Thus, if QCD has a first order phase transition, a second peak at $\Delta \phi=1.4$ from the trigger particle should be observed. The experimental correlation functions [21] have a minimum at this angle which indicates that the phase transition cannot be first order. Better theoretical and experimental understanding is needed before drawing such a strong conclusion.

\section{Conclusions}

In this work we have studied the hydrodynamical response of the quark gluon plasma formed at RHIC to the passage of an energetic particle. We have assumed that significant part of the energy lost by the parton is thermalized and incorporated into the hydrodynamical evolution of the system. We have also shown that the response of the medium is not unique.

By studying the modified flow fields far away from the particle, where the linearized approximation is applicable, we have identified two excitation mechanisms: isentropic excitations, where significant entropy is not produced, and non isentropic excitation, where it is. In the former only sound waves are formed while in the later also the diffuson mode is excited. 
We have also studied the effect that this induced field has on the dihadron distribution associated with a jet. If the excitation is isentropic, large angle correlations at $\Delta \phi=\pi-1.2$ appear as a result of the Mach cone formation which agree with experimental results [21, 22]. Even though in a static medium the required value of the energy loss is large $(d E / d x \approx 12 \mathrm{GeV} / \mathrm{fm})$, expansion effects reduce the necessary loss to $d E / d x \approx 1.5 \mathrm{GeV} / \mathrm{fm}$

Expansion effects are also the responsible for the change of the speed of sound along the matter evolution. If the QCD phase transition is first order (vanishing speed of sound at the mixed phase), then reflected waves should appear, leading to a peak in the correlation functions at $\Delta \phi=1.4$ with respect to the trigger particle. This correlation is not observed. If the appearance of the reflected wave resists further tests, the non observation of this peak implies that the QCD phase transition cannot be first order. However, further work is needed before drawing such a strong conclusion.

Finally, we would like to mention that conical flow has been also found within the context the AdS/CFT correspondence. In [40] the modification of the stress tensor of a $\mathscr{N}=4 S U S Y$ plasma by a high energy particle is studied at strong coupling. It is found that the high energy particle excites sound and leads to the formation of a Mach front. This is remarkable, since it is a fully dynamical calculation, in which no assumption about thermalization nor hydrodynamical behavior is made. These results are very encouraging and further computations within this context may shed light about the details of the jet medium interactions at RHIC.

\section{Acknowledgments}

The result here presented were done in collaborators E. V. Shuryak and D. Teaney. This work was supported by the Director, Office of Science, Office of High Energy and Nuclear Physics, Division of Nuclear Physics, and by the Office of Basic Energy Sciences, Division of Nuclear Sciences, of the U.S. Department of Energy under Contract No. DE-AC03-76SF00098 and by the grants DE-FG02-88ER40388 and DE-FG03-97ER4014.

\section{References}

[1] K. Adcox et al. (PHENIX), Phys. Rev. Lett. 88 (2002) 022301

[2] C. Adler et al. (STAR), Phys. Rev. Lett. 89 (2002) 202301

[3] S. S. Adler et al. (PHENIX), Phys. Rev. Lett. 91 (2003) 072301

[4] J. Adams et al. (STAR), Phys. Rev. Lett. 91 (2003) 172302

[5] J. D. Bjorken, FERMILAB-PUB-82-059-THY

[6] M. Gyulassy, I. Vitev, X. N. Wang and B. W. Zhang, arXiv:nucl-th/0302077.

[7] A. Kovner and U. A. Wiedemann, arXiv:hep-ph/0304151.

[8] R. Baier, Y. L. Dokshitzer,A. H. Mueller,S. Peigne and D. Schiff, Nucl. Phys. B 483 (1997) 291

[9] S. Wicks, W. Horowitz, M. Djordjevic and M. Gyulassy, arXiv:nucl-th/0512076.

[10] E. V. Shuryak and I. Zahed arXiv:hep-ph/0406100

[11] D. Teaney, J. Lauret and E. V. Shuryak, arXiv:nucl-th/0110037. 
[12] P. F. Kolb, U. W. Heinz, P. Huovinen, K. J. Eskola and K. Tuominen, Nucl. Phys. A 696, 197 (2001) [arXiv:hep-ph/0103234].

[13] S. S. Adler et al. [PHENIX Collaboration], Phys. Rev. Lett. 91, 182301 (2003) [arXiv:nucl-ex/0305013].

[14] K. H. Ackermann et al. [STAR Collaboration], Phys. Rev. Lett. 86, 402 (2001) [arXiv:nucl-ex/0009011].

[15] D. Teaney, Phys. Rev. C 68, 034913 (2003) [arXiv:nucl-th/0301099].

[16] G. Policastro, D. T. Son and A. O. Starinets, Phys. Rev. Lett. 87, 081601 (2001) [arXiv:hep-th/0104066].

[17] E. V. Shuryak and I. Zahed, Phys. Rev. C 70, 021901 (2004) [arXiv:hep-ph/0307267].

[18] H. Stoecker, Nucl. Phys. A 750, 121 (2005) [arXiv:nucl-th/0406018].

[19] J. Casalderrey-Solana, E. V. Shuryak and D. Teaney, arXiv:hep-ph/0602183.

[20] J. Casalderrey-Solana, E. V. Shuryak and D. Teaney, J. Phys. Conf. Ser. 27, 22 (2005) [arXiv:hep-ph/0411315].

[21] S. S. Adler et al. (PHENIX Collaboration), [arXiv:nucl-ex/0507004]

[22] J . Adams et al. (STAR Collaboration), Phys. Rev. Lett. 95 (2005) 152301

[23] J. Casalderrey-Solana and E. V. Shuryak, arXiv:hep-ph/0511263.

[24] L. M. Satarov, H. Stoecker and I. N. Mishustin, Phys. Lett. B 627, 64 (2005) [arXiv:hep-ph/0505245].

[25] T. Renk and J. Ruppert, Phys. Rev. C 73, 011901 (2006) [arXiv:hep-ph/0509036].

[26] A. K. Chaudhuri and U. Heinz, Phys. Rev. Lett. 97, 062301 (2006) [arXiv:nucl-th/0503028].

[27] G. L. Ma et al., arXiv:nucl-th/0601012.

[28] J. Ruppert and B. Muller, Phys. Lett. B 618, 123 (2005) [arXiv:hep-ph/0503158].

[29] V. Koch, A. Majumder and X. N. Wang, Phys. Rev. Lett. 96, 172302 (2006) [arXiv:nucl-th/0507063].

[30] A. Majumder and X. N. Wang, Phys. Rev. C 73, 051901 (2006) [arXiv:nucl-th/0507062].

[31] I. M. Dremin, Nucl. Phys. A 767, 233 (2006) [arXiv:hep-ph/0507167].

[32] I. Vitev, Phys. Lett. B 630, 78 (2005) [arXiv:hep-ph/0501255].

[33] A. D. Polosa and C. A. Salgado, arXiv:hep-ph/0607295.

[34] C. B. Chiu and R. C. Hwa, arXiv:nucl-th/0609038.

[35] L. D. Landau and E. M. Lifshitz, Fluid Mechanics

[36] K. J. Eskola, H. Honkanen, C. A. Salgado and U. A. Wiedemann, Nucl. Phys. A 747, 511 (2005) [arXiv:hep-ph/0406319].

[37] L. D. Landau, E. M. Lifshitz, Mechanics, Pergamon Press, 1976

[38] C. M. Hung and E. V. Shuryak, Phys. Rev. C 57, 1891 (1998) [arXiv:hep-ph/9709264].

[39] G. M. Welke, R. Venugopalan and M. Prakash, Phys. Lett. B 245, 137 (1990).

[40] J. J. Friess, S. S. Gubser, G. Michalogiorgakis and S. S. Pufu, arXiv:hep-th/0607022. 